\title{
New or Noteworthy Trees form Micronesia XV
}

\author{
By
}

\section{Ryôzô Kanehira}

With 6 Text-Figures

Received May 5, 1935

A third large collection of Pandanus has been made in Micronesiain Jaluit, Kusai, Ponape and Yap, through the cooperation of various Government officials. Six of the species represented are apparently undescribed. Up to the present time, a total of 47 species of Pandanus are recorded from Micronesia; of these 40 belong to the section Keura, 5 to the section Bryantia, while the section Lophostigma and Hombronia are represented by a single species each.

(154) Pandanus duriocarpoides Kanehira sp. nov. ( $(K e u r a)$

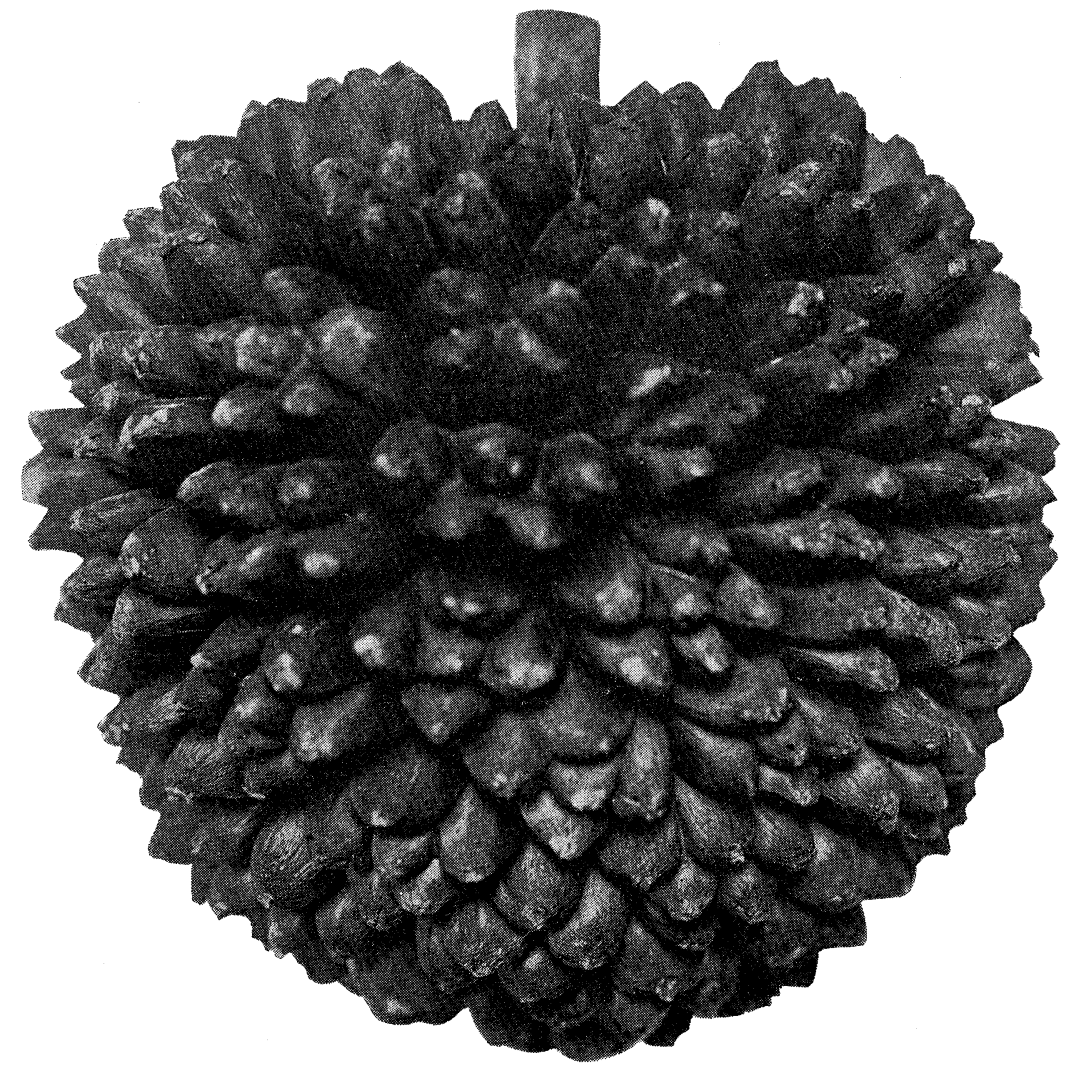

Fig. 37. Pandanus duriocarpoides KANEHIRA 
Syncarpiis solitariis, pendulis, subglobosis, haud oblongis, $18 \mathrm{~cm}$ diametro, phalangiis circiter 90 , obovoideis, $70 \mathrm{~cm}$ longis, $3-5 \mathrm{~cm}$ latis, parte $1 / 3$ superiore libera prismatica, vertice subtruncato, basi longe acuminatis, loculis 5-7, plerumque 5 vel 6 , subaequalibus, irregulariter dispositis, profunde sulcatis, pyramidatis, pyramidis $10 \mathrm{~mm}$ longis, basi usque ad $1.5 \mathrm{~cm}$ latis, stigmatibus magnis, oblique hippocrepiformibus. Endocarpium osseum, in parte centrali positum, superum pyramidatum, margine paullo protruso, inferum subtruncatum, rimosum. Mesocarpium superum cavernosum, cavernis lacunosis, exterioribus fusiformibus, circiter $22 \mathrm{~mm}$ longis, mesocarpium inferum fibrosum.

Syncarps composed of about 90 obovoid phalanges which are so closely arranged that it is impossible to distinguish the individual phalanges from the outside. The phalanges are polygonal in cross section, their sides deeply sulcate, the locules subequal, irregularly arranged, pyramidal. The endocarp is situated in the median part of the phalange and slightly protrudes at margin.

No. 3497! 'Tomil, Yap, near the Wireless Station, Jan. 17, 1935. Collected by the Yap District Office.

The present species resembles Pandanus duriocarpus Martell in appearance but its syncarp and phalanges are much larger and locules are in fewer number. It is also near Pandanus Volkensii Kanehira, but differs from it by its globose syncarps and acute locules.

\section{(155) Pandanus Eyesyes Kaneirira sp. nov. (Keura)}

Syncarpiis oblongis, $30 \mathrm{~cm}$ longis, $24 \mathrm{~cm}$ latis, phalangiis circiter 110, obovoideis vel claviformibus, $10-11 \mathrm{~cm}$ longis, $5 \mathrm{~cm}$ latis, plus-minusve lateraliter compressis, vertice subplano, infra medio sensim angustatocaudatis, longeque cuneatis, parte $1 / 3$ superiore libera, loculis 8 vel 9 , latiusculis, pentagonis, pyramidatis, $5-6 \mathrm{~mm}$ longis, exterioribus majoribus, subconcentrice dispositis, sulcis interlocularibus profundis, stigmate apicali parvo, subobliquo, hippocrepiformi. Endocarpium osseum, exiguum, in partibus superioribus phalangiorum situm, cum mesocarpio supero transverse intermixtum, mesocarpium superum medullosum, paullo carvernosum, usque ad $3 \mathrm{~cm}$ longum, inferum fibrosum, 5-6 cm longum.

Synearps apparently solitary, pendulous, composed of about 110 obovoid or club-shaped, $11 \mathrm{~cm}$ long, $5 \mathrm{~cm}$ wide, laterally compressed phalanges, their shoulders rounded, their apices subtruncate, the marginal locules somewhat divergent. Endocarp very scanty or thin-walled surrounding the seeds.

No. 3503 Kusai Island, Dec. 1934, YosidA, locally known as "eyesyes" (Kusai). 
In first sight, the plant resembles Pandanus pedunculatus $\mathrm{R}$. BR., which occurs in the Solomon and Bismarck Islands and in Australia, but it is readily distinguished by its very characteristic endocarps.

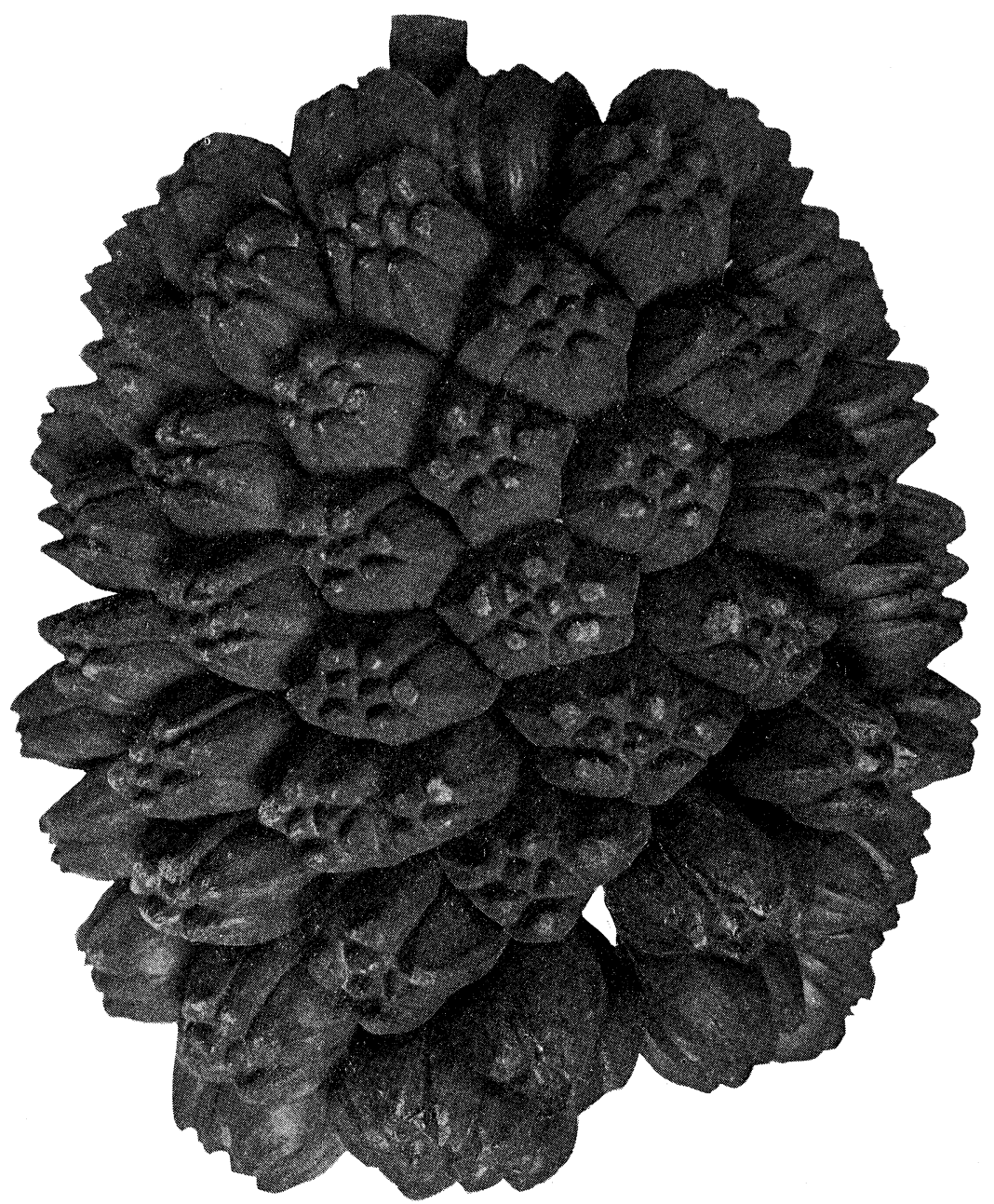

Fig. 38. Pandanus Eyesyes KANEHIRA

(156) Pandanus insularis Kanehira sp. nov. ( $\$$ Keura)

Syncarpiis ut videtur solitariis, pendulis, subglobosis, fere $22 \mathrm{~cm}$ longis, $16 \mathrm{~cm}$ latis, phalangiis numerosis, obovoideis, $6.5 \mathrm{~cm}$ longis, $4.5 \mathrm{~cm}$ crassis, superne rotundatis, in partibus inferioribus cuneato-angustatis, vertice leviter rotundato, loculis numerosis, 8-12, interdem usque ad 16, subaequalibus, latiusculis, basi circiter $6-8 \mathrm{~mm}$ latis, paullo elevatis vel subplanis, sulcis haud profundis, stigmate suberecto, minuto. Endocarpium 


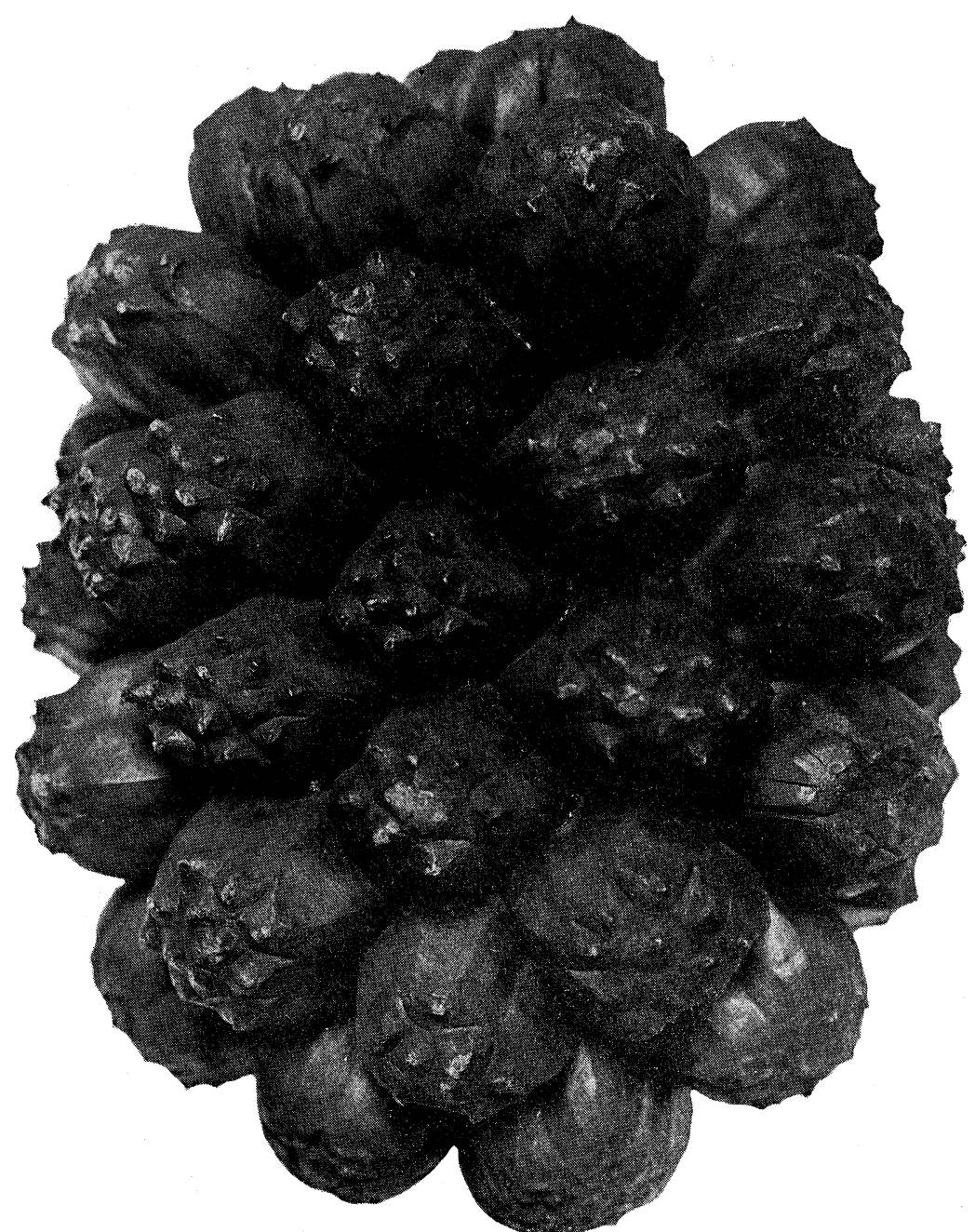

Fig. 39. Pandanus insularis KANEHIRA

osseum, in parte centrali locatum, superne rotundato-pyramidatum, inferne subtruncatum, $2.5 \mathrm{~cm}$ spissum. Mesocarpium superum cavernosum, subaequale, exterioribus $2 \mathrm{~cm}$ Iongis, mesocarpium inferum fibrosum, $3 \mathrm{~cm}$ longum.

Syncarps composed of about 76 obovoid phalanges, their upper parts rounded, the bases gradually narrowed, locules numerous, slightly convex, shallowly sulcate or almost plane, stigma subvertical, minute, about $1.5 \mathrm{~mm}$ wide. Endocarps osseus, the upper parts irregularly pyramidal, the lower parts subtruncate, the upper mesocarps pithy, distinctly chambered, chambers nearly equal-sized.

No. 3504, Kusai, collected by a government official stationed at the 
Kusai District Office, Dec. 1934, locally known as "meu kosa” (Kusai).

This may be allied to Pandanus Okamotoi KaneHira from Palau, but it is easily distinguished from it by its oblong syncarps and its shallow and indistinct sulci.

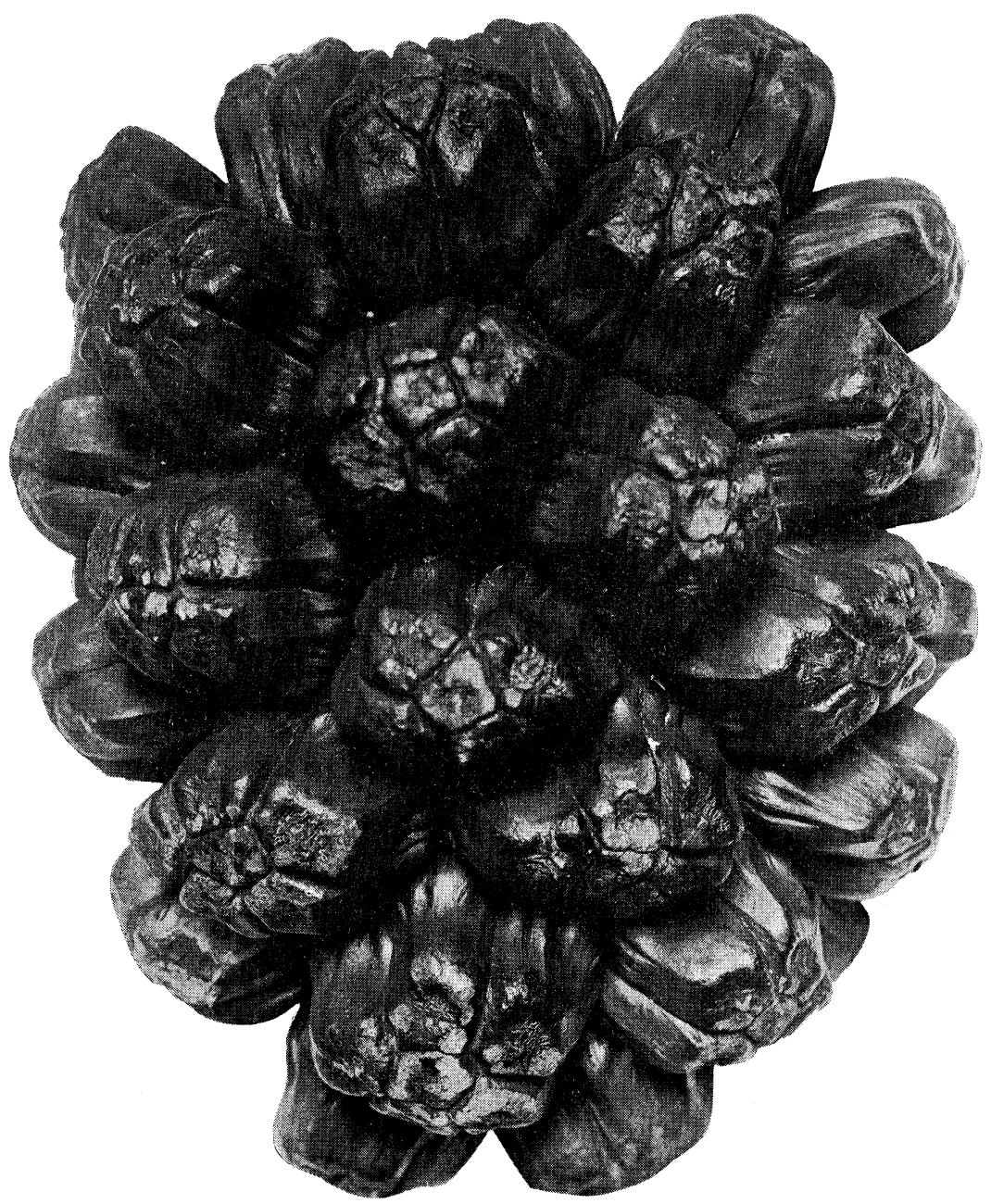

Fig. 40. Pandanus Lakatwa KANEHIRA

(157) Pandanus Lakatwa Kanehira sp. nov. ( $(K e u r a)$

Syncarpiis solitariis, pendulis, oblongis, $30 \mathrm{~cm}$ longis, $23 \mathrm{~cm}$ diametro. Phalangiis obovoideis, $10 \mathrm{~cm}$ longis, $5.5 \mathrm{~cm}$ crassis, vertice subtruncato, parte superiore libera, pyramidata, basi perspicue dilatatis, partibus inferioribus phalangiorum abrupte longeque angustatis. Loculis 5 saepe 6 , concentrice connatis, exterioribus valde majoribus, usque ad $2-2.5 \mathrm{~cm}$ latis, 
aliquando concavis haud elevatis, sulcis non profundis sed latissicule separatis, sigmate sicco immerso, brevi, plus munisve lobato. Endocarpium in dimido superiore locatum, superne irregulariter pyramidatum, inferne subtruncatum, usque ad $3 \mathrm{~cm}$ spissum, mesocarpiis superiores cavernae, exteriores latissimae usque ad $2 \mathrm{~cm}$ longae, interiores parvae. Mesocarpium inferum fibrosum circiter $6 \mathrm{~cm}$ longum.

Syncarps composed of about 67 obovoid phalanges, their free parts pyramidal, the apical parts subtruncate, the bases prominently swollen. Locules usually 5, nearly concave, not elevated, stigma immersed when dry, small, slightly bilobed.

No. 3516 Jaluit, Dec. 1934. Collected by a government official. Locally known as "lakatwa" (Jaluit).

The nearest alliance is no doubt with Pandanus laticanaliculatus KANEHIRA but the present species has truncate phalanges, the free portion of which are pyramidal and the number of locules are fewer.

(158) Pandanus obliquus Kanemrea sp. nov. ( $\$$ Keura)

Syncarpiis ut videtur solitariis, pendulis, oblongis, circiter $42 \mathrm{~cm}$ longis, $21 \mathrm{~cm}$ latis, phalangiis obpyriformibus, plus minusve obliquis, $10-11 \mathrm{~cm}$ longis, 5-6, interdeum $7 \mathrm{~cm}$ diametro, vertice subtruncato, inferne abrupte longeque angustatis, faciebus longitudinaliter costulatis, fere in quarta superiore liberis, loculis 6 vel 7, latiusculis, inaequalibus, irregulariter connatis, pyramidatis, $10-15 \mathrm{~mm}$ longis, basi usque ad $20 \mathrm{~mm}$ latis, stigmate excentrico, auriculiformi, subverticali. Endocarpiüum osseum, superne in parte centrali pyramidatum, inferne truncato-rimosum, margine leviter protruso, mesocarpium superum cavernosum, cavernis fere inaequalibus, exterioribus latissimis, usque ad $3.5 \mathrm{~cm}$ longis, mesocarpium inferum fibrosum, $5 \mathrm{~cm}$ longum.

Syncarps composed of about 105 obliquely obpyriform phalanges, their sides somewhat rounded and longitudinally and profoundly sulcate. Locules unequal-sized and rather irregularly arranged, prominently notched on the inner side.

No. 3525! Jaluit, collected by government officials stationed at the Jaluit District Office, Dec. 1934, locally known as "lajokorer" (Jaluit).

The species is characterized by its very large, oblong syncarps, its somewhat oblique phalanges, and its unequally sized and irregularly arranged locules.

(159) Pandanus tomilensis Kanehira sp. nov. ( $\$$ Keura)

Syncarpiis subglobosis vel ellipticis, $16 \mathrm{~cm}$ diametro, phalangiis obpyriformibus, latiusculis, $7 \mathrm{~cm}$ longis, $3-4 \mathrm{~cm}$ latis, faciebus in partibus superioribus rotundatis, vertice subtruncato, basi sensim cuneatis, partibus 1/3 


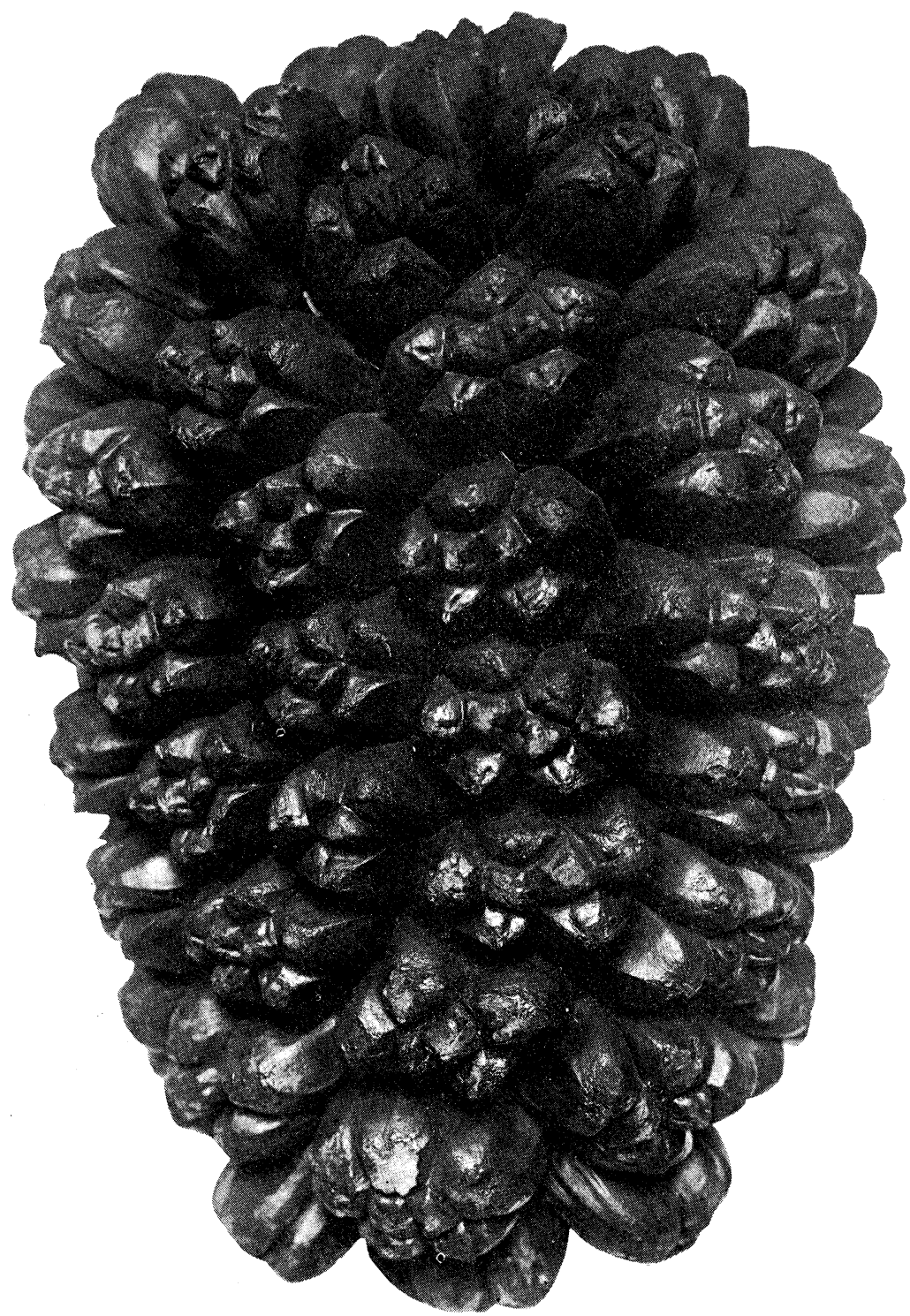

Fig. 41. Pandanus obliquus Kanemira

superioribus liberis, loculis 4-6, plerumque 5, subaequalibus, exterioribus paullo majoribus, irregulariter connatis, paullo convexis, sulei haud profundis, stigmatibus apicalis, plus minusve divergentibus. Endocarpium osseum, fere in medio positum, superum pyramidatum, margine perspicue protruso, inferum rimosum, mesocarpium superum cavernosum, cavernis medullosis, exterioribus majoribus, usque ad $2.5 \mathrm{~cm}$ longis, inferum fibrosum, circiter $3 \mathrm{~cm}$ longum. 


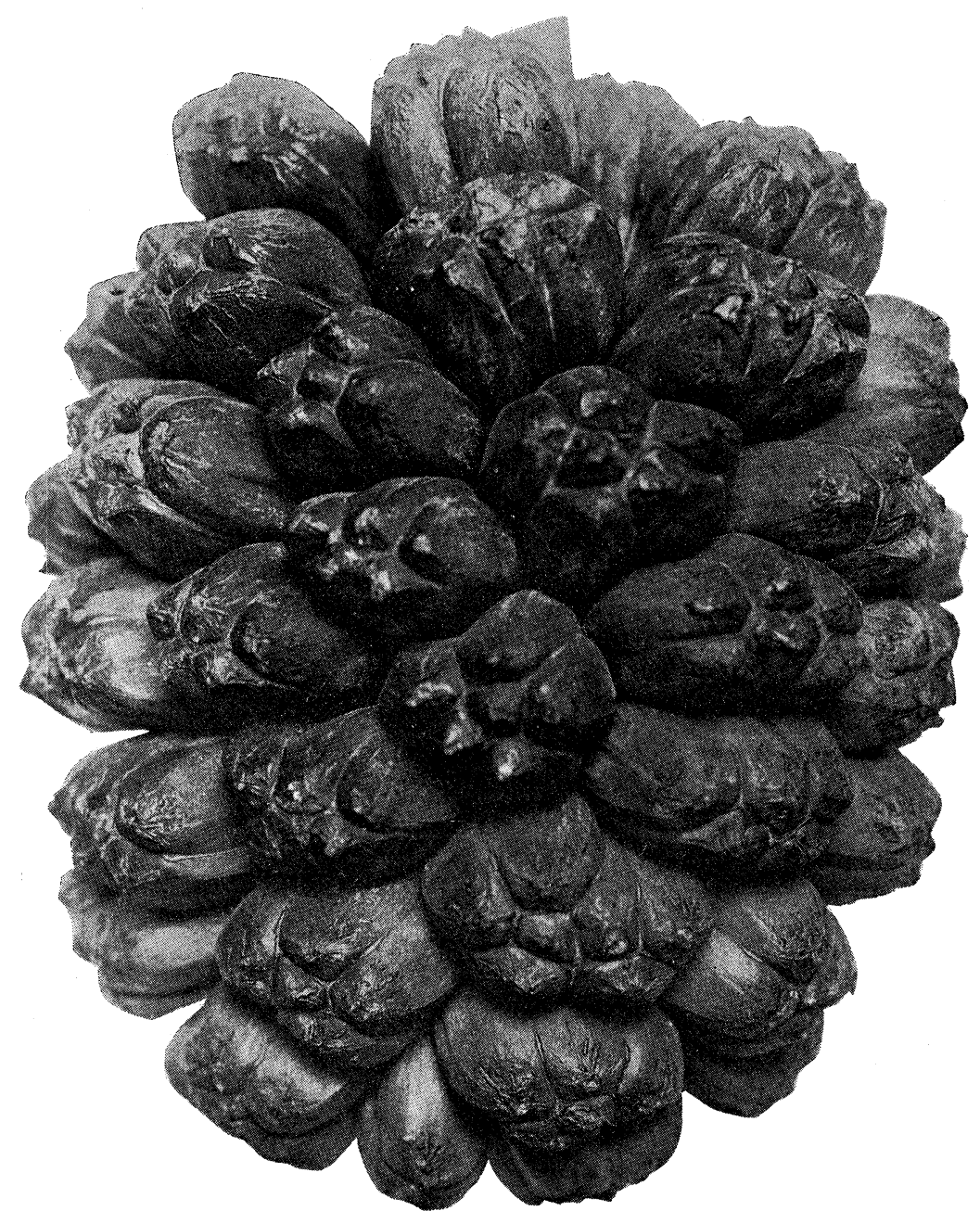

Fig. 42. Pandanus tomilensis KANEHIRA

Syncarps composed of about 90 phalanges. Phalanges obpyriform. laterally compressed, the apex subtruncate, locules convex or nearly plane excepting those at the base of syncarp; sulci between locules very shallow, stigma suberect, usually surrounded by a corky substance.

No. 3498 Tomil, Yap, near the Wireless Station, Jan. 17, 1934. Collected by a Yap District officer.

This species manifestly allied to Pandanus guamensis MARTELLI but it is distinguished by its subequal locules. It also resembles Pandanus fragrans GaUdich., but it is easily distinguished from the latter by its obovoid phalanges and more numerous locules. 